\title{
Isolated Lacrimal Gland Enlargement in Thyroid-Related Orbitopathy
}

\author{
Tal Yahalomi ${ }^{a}$ Joseph Pikkel ${ }^{a}$ Roee Arnon ${ }^{a}$ Eugene Soikher ${ }^{b}$ \\ Avi Rubinov ${ }^{a}$ \\ aDepartment of Ophthalmology, Samson Assuta Ashdod Hospital, Faculty of Health \\ Sciences, Ben-Gurion University of the Negev, Beersheba, Israel; bepartment of Radiology, \\ Samson Assuta Ashdod Hospital, Faculty of Health Sciences, Ben-Gurion University of the \\ Negev, Beersheba, Israel
}

\section{Keywords}

Lacrimal gland enlargement · Thyroid-related orbitopathy · Eye disease · Eyelid · Imaging

\begin{abstract}
Thyroid-related orbitopathy (TRO) is considered to be an inflammatory autoimmune disorder that commonly presents with extraocular muscle and retrobulbar fat enlargement. Lacrimal gland enlargement in TRO has been reported in the past in association with other radiological findings. Our case represents an isolated lacrimal gland enlargement as a presenting radiological finding in an euthyroid patient showing elevation of thyroid-stimulating immunoglobulin. After ruling out possible pathologies such as a monoclonal process, lacrimal epithelial tumor, or a nonspecific orbital inflammation, the patient underwent orbital decompression, serial botulinum toxin injections, and upper eyelid-retraction surgical correction. After two years of follow-up, the patient developed new-onset diplopia; consequent imaging showed extraocular muscle enlargement which was not present on previous imaging.
\end{abstract}

\section{Introduction}

The incidence rate of thyroid-related orbitopathy (TRO) is 3:100,000 in men and $16: 100,000$ in women with up to $5 \%$ of patients showing severe disease accompanied with sight-threatening vision loss due to corneal ulceration or optic nerve compression [1]. Lacrimal gland involvement in TRO has been demonstrated in various clinical and radiologic reports $[2,3]$. One study of 128 patients demonstrated a statistically significant enlarged 
Yahalomi et al.: Isolated Lacrimal Gland Enlargement as a Presenting Sign in ThyroidRelated Orbitopathy

lacrimal gland in TRO with smoking identified as an isolated risk factor for glandular enlargement [4].

In TRO, lacrimal gland tissue is suggested to be involved in the inflammatory response, causing lacrimal enlargement and later aqueous tear deficiency in correlation with a decrease in quantity and composition of tears produced [5-9]. In addition, a study of 71 patients concluded that compressive optic neuropathy in TRO was correlated with an enlarged, prolapsed lacrimal gland [8].The unique presentation of TRO as mere isolated lacrimal gland enlargement was only presented once by Khu and Freedman [10].

\section{Case Presentation}

A 52-year-old-female was referred to our clinic due to a right eye proptosis present for 2 months (Fig. 1a) without diplopia or other complaints. Her ophthalmologic examination showed right proptosis and right upper eyelid retraction. Superior marginal reflex distance was $5 \mathrm{~mm}$ on the right and $3.5 \mathrm{~mm}$ on the left. Her visual acuity was $20 / 20$ in both eyes, ocular motility was full, her slit lamp and fundus exams were unremarkable in both eyes, Ishiara color plates were $12 / 12$ in both eyes, pupillary responses were normal, and she had $4 \mathrm{~mm}$ of right-sided proptosis. Humphrey visual fields were normal bilaterally. Ocular ultrasonography and computerized tomography of the orbits did not reveal any ocular or adnexal abnormalities, and thyroid-stimulating hormone was within normal limits.

Later in her follow-up, she had developed new-onset binocular horizontal diplopia. A laboratory workup was performed which included repeated thyroid function tests (tri-iodothyronine 3 and 4, and thyroid-stimulating hormone) and a rheumatic panel comprising antinuclear antibody, antineutrophil cytoplasmic antibody, rheumatic factor, and angiotensinconverting enzyme. All results were within normal limits. MRI scan of the orbits was performed. The only pathologic finding revealed by the scan was irregular enlargement of the right lacrimal gland (Fig. 2). No other radiological abnormalities were detected. The patient subsequently underwent anterior orbitotmy with lacrimal gland incisional biopsy that revealed normal acinar glandular configuration with periacinar lymphocytic infiltration. Immunohistochemistry was negative for IgG4-related orbital disease and for a monoclonal process. The patient received a course of oral prednisone, starting from $60 \mathrm{mg}$ daily, with a slow taper over 6 weeks without any change in diplopia or proptosis. Graves' ophthalmopathy

Fig. 1. a Patient prior to orbital decompression. b Following decompression surgery, botulinum toxin injection, and eyelid repair for treatment of upper eyelid retraction.
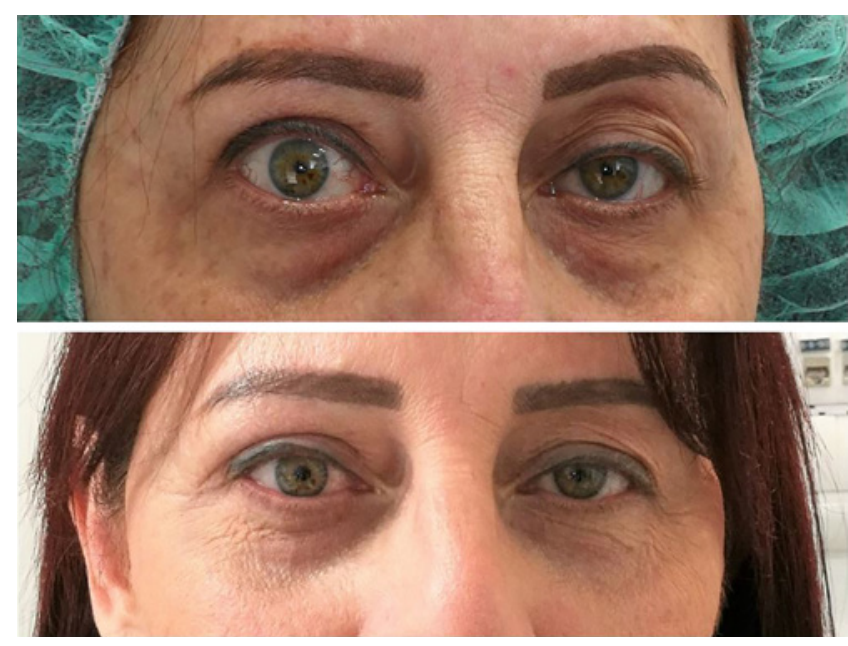
Yahalomi et al.: Isolated Lacrimal Gland Enlargement as a Presenting Sign in ThyroidRelated Orbitopathy

Fig. 2. a Coronal T1-weighted contrastenhanced MR scan of orbits demonstrates irregular enlargement of the right lacrimal gland. b Normal extraconal muscles.
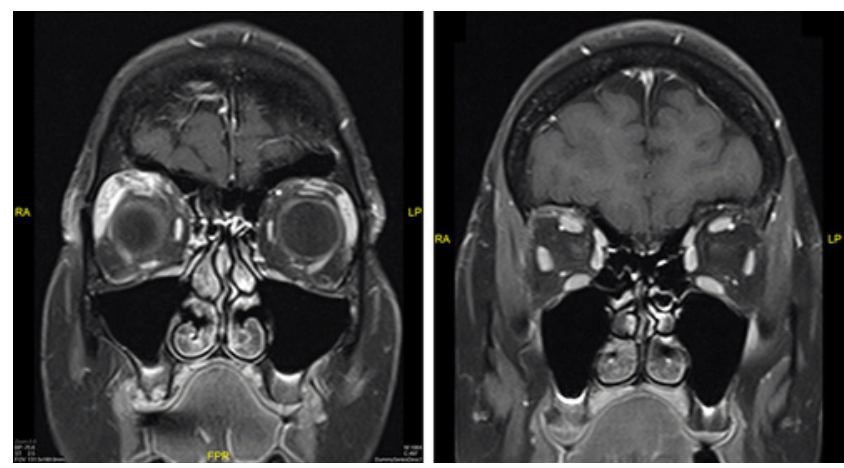

Fig. 3. a Coronal computed tomography of the brain without contrast enhancement at presentation demonstrates normal extraconal muscles. b Coronal computed tomography of the brain without contrast enhancement 2 years after presentation demonstrates irregular enlargement of the left inferior rectus.
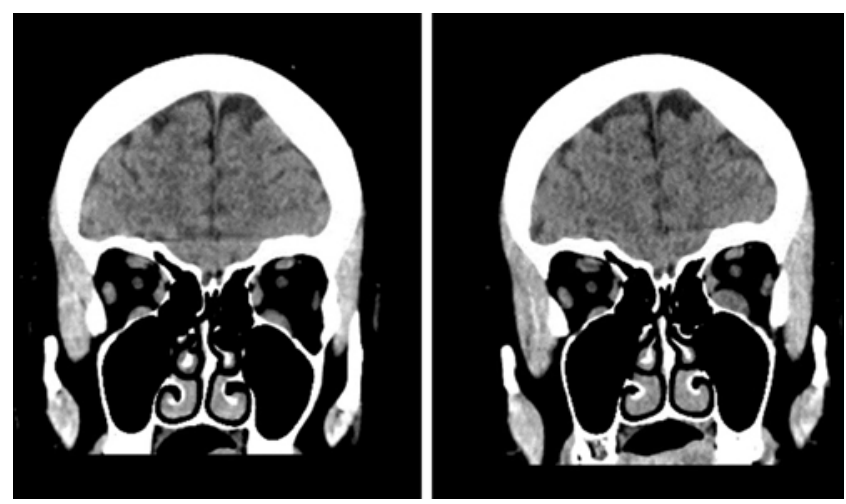

was still suspected, and a thyroid-stimulating immunoglobulin (TSI) was performed showing a level of $200 \%$ (normal TSI $<140 \%$ ) pointing the diagnosis toward TRO.

A year following her presentation, after reassurance of clinically stable TRO, she underwent right orbital decompression. This included a deep lateral wall burring and a further debulking and biopsy of the remaining orbital part of the lacrimal gland, which showed the same reactive changes as found previously. Following the decompression, serial Botox injections and an eyelid retraction repair were performed for the treatment of right upper eyelid retraction. Following surgery, diplopia symptoms were resolved and the patient was satisfied with the esthetic result (Fig. 1b).

One year following the decompression surgery, the patient complained of a new-onset diplopia, and a repeated computed tomography showed a new enlargement of extraocular muscles, this time in the left eye (Fig. 3). Since the disease had been reactivated, the patient was put on intravenous methylprednisolone on a weekly dose for 12 weeks. Currently she is still receiving that treatment, and the diplopia has stabilized without appearance of new TROrelated soft tissue signs.

\section{Discussion}

TRO serves as a relatively common disorder presented in ophthalmic clinics and is mostly a clinical diagnosis. In most cases, the clinical signs and laboratory studies for thyroid function and imaging help to ascertain the diagnosis. The most familiar clinical signs for TRO include proptosis, restrictive myopathy, eyelid retraction, and conjunctival edema, while the classical radiological sign are extraocular muscle enlargement. Lacrimal gland enlargement in TRO is little discussed albeit clinical and radiologic reports [2, 3]. 
Yahalomi et al.: Isolated Lacrimal Gland Enlargement as a Presenting Sign in ThyroidRelated Orbitopathy

Isolated lacrimal gland involvement in TRO is not sufficiently demonstrated in the literature despite one case presented by Khu and Freedman [10]. We present a case of a 52-year-old female with isolated lacrimal gland enlargement as a presenting sign of TRO. The lacrimal gland enlargement was the sole radiological sign for two years of follow-up until a newly discovered radiological signs of fusiform enlargement of the extraocular muscles. The markedly elevated TSI levels assisted us in the laboratory diagnosis of TRO, similar to the case presented by Khu and Freedman [10]. As TRO is primarily a clinical diagnosis, it is important to discuss the natural history of the disease and the possibility of lacrimal glandular enlargement as the presenting radiological finding.

\section{Statement of Ethics}

Written informed consent was obtained from the patient for publication of this case report and any accompanying images. This report is adhered to the ethical principles outlined in the Declaration of Helsinki as amended in 2013.

\section{Conflict of Interest Statement}

The authors have no conflicts of interest to declare

\section{Funding Sources}

No funding or grant support.

\section{Author Contributions}

The following authors had substantial contributions to the conception and the design of the work, drafting the work, and revising it: A.R., T.Y., J.P., R.A., and E.S.: All contributors fulfill the ICMJE criteria for authorship.

\section{References}

1 Bahn RS. Graves' ophthalmopathy. N Engl J Med. 2010;362(8):726.

2 Trokel SL, Jakobiec FA. Correlation of CT scanning and pathologic features of ophthalmic Graves' disease. Ophthalmology. 1981;88(6):553-64.

3 Rootman J. Diseases of the orbit: a multidisciplinary approach. Philadelphia, PA: Lippincott Williams \& Wilkins; 2003.

4 Harris MA, Realini T, Hogg JP, Sivak-Callcott JA. CT dimensions of the lacrimal gland in Graves orbitopathy. Ophthal Plast Reconstr Surg. 2012;28(1):69-72.

5 Huang D, Luo Q, Yang H, Mao Y. Changes of lacrimal gland and tear inflammatory cytokines in thyroid-associated ophthalmopathy. Invest Ophthalmol Vis Sci. 2014;55(8):4935-43.

6 Khalil HA, de Keizer RJ, Kijlstra A. Analysis of tear proteins in Graves' ophthalmopathy by high performance liquid chromatography. Am J Ophthalmol. 1988;106(2):186-90.

7 Khalil HA, de Keizer RJ, Bodelier VM, Kijlstra A. Secretory IgA and lysozyme in tears of patients with Graves' ophthalmopathy. Doc Ophthalmol. 1989;72(3-4):329-34.

8 Nugent RA, Belkin RI, Neigel JM, Rootman J, Robertson WD, Spinelli J, et al. Graves orbitopathy: correlation of CT and clinical findings. Radiology. 1990;177(3):675-82.

9 Wall J. Extrathyroidal manifestations of Graves' disease. J Clin Endocrinol Metab. 1995;80(12):3427-9.

$10 \mathrm{Khu}$ J, Freedman KA. Lacrimal gland enlargement as an early clinical or radiological sign in thyroid orbitopathy. Am J Ophthalmol Case Rep. 2017;5:1-3. 